\title{
A PROSPECTIVE STUDY OF THE USEFULLNESS OF UPPER LIP BITE TEST IN COMBINATION WITH STERNO- MENTAL DISTANCE, THYRO-MENTAL DISTANCE AND INTER-INCISOR DISTANCE AS PREDICTOR OF EASE OF LARYNGOSCOPY
}

\author{
Chandita Konwar1 , Nabarup Dutta Baruah², Priyam Saikia3, Anulekha Chakrabartty ${ }^{4}$ \\ ${ }^{1}$ Assistant Professor, Department of Anaesthesiology \& Critical Care, Gauhati Medical College and Hospital. \\ ${ }^{2}$ Registrar, Department of Anaesthesiology \& Critical Care, Gauhati Medical College and Hospital. \\ ${ }^{3}$ Assistant Professor, Department of Anaesthesiology \& Critical Care, Gauhati Medical College and Hospital. \\ ${ }^{4}$ Retired Professor, Department of Anaesthesiology \& Critical Care, Gauhati Medical College and Hospital.
}

\begin{tabular}{l} 
ABSTRACT \\
\hline BACKGROUND \\
Upper Lip Bite Test (ULBT) has been evaluated as a simple bedside test to predict the grade of laryngeal visualisation. As the \\
utility of this test is not yet evaluated in patients from this geographical location of India, we intend to investigate whether the \\
combination of the ULBT classification with Sternomental Distance (SMD), Thyromental Distance (TMD), and Inter-Incisor \\
Distance (IID) to predict easy laryngoscopy and compared with each test alone.
\end{tabular}

\section{METHODS}

In a prospective study, 200 patients scheduled for elective surgery were selected randomly and enrolled in the study. During preoperative visit, the airways were assessed and ULBT class, SMD, TMD, and IID measured. Class III ULBT, SMD $<12.5 \mathrm{~cm}$, TMD $<6.5 \mathrm{~cm}$ and IID $<4.0 \mathrm{~cm}$ was defined as a predictor of "Difficult Visualisation of Larynx (DVL)." After induction of Anaesthesia and skeletal muscle relaxation, grade of direct laryngoscopic view was determined. Cormack and Lehane grades 3 and 4 defined as DVL.

\section{RESULTS}

The prevalence of difficult intubation was $11 \%(\mathrm{n}-22)$. Specificity and accuracy of the ULBT were significantly higher than TMD, SMD, and IID individually (Specificity was 94.3\%, 89.8\%, 87.6\%, 88.0\% respectively and accuracy was 91.5\%, 87.0\%, 86.0\% and 81.5\% respectively). The combination of the ULBT with SMD provided the highest sensitivity (50.0\%).

\section{CONCLUSION}

Our study demonstrates that the Upper Lip Bite Test (ULBT) is the best predictive test for difficult visualization of larynx among the predictive tests evaluated and combination of the other tests with ULBT improves the ability their specificity.

\section{KEYWORDS}

Airway Management; Inter-Incisor Distance; Laryngoscopy; Predictive Value of Tests; Sternomental Distance; Thyromental Distance; Upper Lip Bite Test.

HOW TO CITE THIS ARTICLE: Chandita Konwar, Nabarup Dutta Baruah, Priyam Saikia, Anulekha Chakrabartty. "A Prospective Study of the usefullness of Upper Lip Bite Test in Combination with Sternomental Distance, Thyromental Distance and Inter-Incisor Distance as Predictor of Ease of Laryngoscopy." Journal of Evolution of Medical and Dental Sciences 2015; Vol. 4, Issue 97, December 03; Page: 16286-16289, DOI: 10.14260/jemds/2015/2401

\section{INTRODUCTION}

Airway management in an anaesthetised patient is a major responsibility of an Anaesthesiologist. Failure to intubate the trachea or secure a patent airway, even for a few minutes can lead to catastrophic outcome. The incidence of difficult intubation is estimated to be approximately $1 \%-18 \%{ }^{1}$ Although many bedside preoperative methods of assessment of the airway and their combinations have been evaluated to predict the possibility of difficult airway, there is conflicting data regarding their accuracy.2,3

Financial or Other, Competing Interest: None.

Submission 24-11-2015, Peer Review 25-11-2015

Acceptance 27-11-2015, Published 03-12-2015.

Corresponding Author:

Dr. Chandita Konwar,

House No. 12. Harabala Road, Near

Bora Service, Ulubari, Guwahati-781007,

Assam.

E-mail: chandita3715@yahoo.co.in

DOI:10.14260/jemds/2015/2401
Moreover, many preoperative airway tests are found to have only fair interobserver reproducibility. 4

Khan Z. H., et al. have proposed that the Upper Lip Bite Test (ULBT) could serve as a good predictor of difficult laryngoscopic intubation. ${ }^{2}$ Any test to be universally accepted, it is required to validate it in various populations. Although the utility of ULBT has been evaluated in patients from Western India, no such data is available from the Eastern population. ${ }^{1}$ Therefore, the present study was conducted to find out the validity of ULBT, Thyromental Distance (TMD), Sternomental Distance (SMD) and Inter-Incisor Distance (IID) individually and in combination.

\section{METHODS AND MATERIALS}

This prospective blinded observational study was carried out after obtaining ethical committee clearance (No. MC/233/2013/137) and informed consent from the participants during the period of December 2013 to August 2014. 
Two hundred (200) patients were randomly selected and enrolled in this study. The study population consisted of patients of American Society of Anesthesiology (ASA) class I and II, belonging to either sex of age group of 18-40 years admitted for operation under general anesthesia with endotracheal intubation. Patients with concurrent pregnancy; intraoral, laryngeal or pharyngeal mass; altered head and neck anatomy; and restricted movement of the neck were excluded. Preoperatively, single investigator who is trained in the planned test but not involved in laryngoscopy and intubation examined the patient's airway and collected data.

The SMD was measured in supine position with the head fully extended and with the mouth closed. The straight distance between the upper border of the manubrium sterni and the bony point of the mentum was measured. 5

For measurement of IID, the patient was asked to open his/her mouth and the distance between incisors was obtained. ${ }^{5}$

TMD of the subjects was measured with the head in complete extension. The distance between the laryngeal prominence of the thyroid cartilage and the mental protuberance of the mandible was measured. ${ }^{6}$

ULBT was measured as described by Khan Z. H., et al. ${ }^{2}$ The patients were asked to bite their upper lip and scoring was performed according to the following criteria. ${ }^{2}$

Class I: Lower incisors can bite the upper lip above the vermilion line.

Class II: Lower incisors can bite the upper lip below the vermilion line.

Class III: Lower incisors cannot bite the upper lip.

SMD $\geq 12.5 \mathrm{~cm}$, IID $\geq 4 \mathrm{~cm}$, ULBT class I and II and $\mathrm{TMD} \geq 6.5 \mathrm{~cm}$ was selected as indicator of Easy Visualization of Larynx (EVL).

On the morning of surgery, the patient was shifted to the operation theatre and the standard ASA monitors were attached. After induction of anaesthesia and neuromuscular blockade using a similar anaesthesia protocol, patient's lungs were ventilated with $100 \%$ oxygen and the head was placed on an intubating pillow (8cm in height). Direct laryngoscopy was attempted in the sniffing position by using Macintosh blade no 3. All laryngoscopies were performed by a senior anaesthesiologist with more than two years of experience post qualification. Cormack and Lehane ( $\mathrm{C}$ and $\mathrm{L}$ ) grading was noted without any external laryngeal pressure. ${ }^{7}$ The following is the $\mathrm{C}$ and $\mathrm{L}$ grading used for the purpose of this study: ${ }^{7}$

Grade 1: Visualization of entire laryngeal aperture.

Grade 2: Visualization of only posterior commissure of laryngeal aperture.

Grade 3: Visualization of only epiglottis.

Grade 4: Nonvisualization of even the epiglottis.

The patient's trachea was intubated with appropriate sized endotracheal tube and anaesthesia was maintained. C and L grading of I and II was labelled as EVL, whereas grade III and IV are labelled as Difficult Visualization of Larynx (DVL). ${ }^{2}$

\section{STATISTICAL ANALYSIS}

The data were entered into a Microsoft Excel Worksheet and analyzed using SPSS (Version: 15.1) statistical package. Demographic data are summarized based on their central and dispersion statistical indices. Using a $2 \times 2$ table contingency table, sensitivity, specificity, Positive Predictive Value (PPV),
Negative Predictive Value (NPV) and accuracy were calculated with direct laryngoscopic view as the gold standard. The description of the terms used to measure validity of a diagnostic method is mentioned in Table: 1 . Continous data was analysed with independent samples t-test and a " $p$ " value of less than 0.05 was accepted as indicating statistical significance, whereas categorical variable were compared by Mann-Whiteny U test (Table : 2).

\section{RESULTS AND OBSERVATIONS}

All of the two hundred (200) patients that were approached completed this study. Majority of the patients (117 numbers in total, 58.5\%) were male. Irrespective of the gender, most of the patients (53.8\% of males and $56.6 \%$ of females) were in the age group of 21-30 years of age.

Twenty five patients (12.5\%) had ULBT class III and thirty two (16\%) patients had TMD of $<6.5 \mathrm{~cm}$. The prevalence of SMD of $<12.5 \mathrm{~cm}$ and IID of $<4 \mathrm{~cm}$ was $19 \%$ (Thirty eight numbers in total) and $13.5 \%$ (Twenty seven numbers in total) respectively.

The prevalence of DVL was found to be $11 \%$ (Twenty two among two hundred laryngoscopies). All the patients with DVL could be intubated with the help of external laryngeal manipulation. The central tendency and the dispersion of demographic data and predictors of airway among the EVL and DVL group are presented in the Table: 2 . The performance of ULBT Class III, SMD $<12.5 \mathrm{~cm}$, TND $<6.5 \mathrm{~cm}$ and IID of $4.0 \mathrm{~cm}$ compared to the 'gold standard' of direct visualisation larynx is mentioned in Table: 3 .

As evident from the Table: 4, when considered alone, SMD of $<12.5 \mathrm{~cm}$ and ULBT of class III have the highest sensitivity and specificity in prediction of DVL respectively. Whereas when a combination of two tests was employed, combination of ULBT and SMD had the highest sensitivity. Specificity of combination of ULBT with any other tests was similar (Table: 4).

\section{DISCUSSION}

The fundamental responsibility of an Anaesthesiologist is to maintain adequate gas exchange by managing the airway, which is almost continuously patent. To ease the process of laryngoscopy and intubation, various prospective methods to assess the airway have been developed.

In our study, the prevalence of DVL was $11 \%$. Studies from India and other parts of the world reported similar prevalance. $1,8,9,10$ Prevelances ranging from lower to higher value compared to our observation has been reported in literature. ${ }^{2,9,11}$ Anthropometric differences among the studied population might have led to this difference. ${ }^{9}$

A high sensitivity, PPV and NPV is desirable in a test used to predict difficult intubation. ${ }^{5}$ Our study shows that ULBT Class III has highest accuracy and specificity than other tests when used alone. The predictive value of ULBT of class less than III for easy intubation was higher. One important finding from our study is that the combination of ULBT Class III with other predictors markedly improved their specificity, but failed to increase the sensitivity.

Although we observed that the sensitivity of SMD was marginally higher than ULBT class III, its lower PPV makes it less ideal to predict ease of intubation.

The sensitivity and specificity of ULBT class III in predicting DVL in our population is in agreement of values 
reported in literature.2,5,11 The reported sensitivity among different population varies from $28.2 \%$ to $98.6 \% .^{1,8} \mathrm{NPV}$ value of ULBT of our study is similar to previously reported value, but lower NPV was noted by Wajekar A.S., et al. and Safazi M., et al.1,11 Although Honarmand A., et al. and Safazi M., et al. observed a high PPV; many other researchers have reported far lower value.10,11 The PPV varies widely among different studies. ${ }^{2,5,10,11}$ The accuracy of ULBT in our study population is similar to other reported values.,2,5,8 Although the number of patients enrolled in these studies were limited in a study with large Iranian population, ULBT has also been shown to be superior to variables derived from radiological measurements. 2,3,5,10 However, ULBT was found to be a poor predictor of difficult laryngoscopy in a North American patient population. ${ }^{12}$ These observed discrepancies may be due to the fact that there occurs substantial interobserver variability while measuring SMD, TMD and IID, wheras ULBT is associated with less interobserver variability. 5,8

The parameter that are used to analyze the validity of ULBT are almost in agreement in studies carried out across the world, whereas there are major differences for the values reported for other commonly used test. It must be noted that these studies were carried out in population with diverse anthropometric features. There is high interobserver reliability for ULBT, but there is less than ideal interobserver reliability while measuring many of the other bedside airway assessment methods. 5,8

Combination of ULBT class III most markedly improved the diagnostic accuracy of IID among other parameter under study. NPV of combination of ULBT with other tests was similar to value reported by Khan Z.H., et al. 2009, but we observed a lower sensitivity and higher specificity, PPV and accuracy. ${ }^{5}$ Safazi M., et al. found that combination of ULBT with other parameters for assessing airway increases sensitivity and negative predictive value. ${ }^{11}$ While comparing the PPV and NPV of diagnostic methods, we must also consider the fact that these parameters depends on the prevalence of the disease or outcome in consideration and the prevalence of DVL varies widely among various population. $1,8,9,10$

To conclude, our study demonstrates that the Upper Lip Bite Test (ULBT) is the best predictive test for difficult visualization of larynx among the predictive tests evaluated and combination of the other tests with ULBT improves the ability of their specificity.

There was several limitation of this study. Data were collected from elective surgical patients admitted in single Tertiary Care Centre, who did not have gross airway abnormality. Our conclusion may not be applicable to all subgroups of the general population. The cut-off value of the parameter under evaluation was taken from most frequently quoted values in literature as no such value has been reported for our study population.

\section{REFERENCES}

1. Wajekar AS, Chellam S, Toal PV. Prediction of ease of laryngoscopy and intubation-role of upper lip bite test, modified Mallampati classification, and thyromental distance in various combination. J Family Med Prim Care. 2015 Jan-Mar;4(1):101-5.

2. Khan ZH, Kashfi A, Ebrahimkhani EA. Comparison of the upper lip bite test (A simple new technique) with modified Mallampati classification in predicting difficulty in endotracheal intubation: a prospective blinded study. Anesth Analg. 2003 Feb;96(2):595-9.

3. Khan ZH, Arbabi S. Diagnostic value of the upper lip bite test in predicting difficulty in intubation with head and neck landmarks obtained from lateral neck x-ray. Indian J Anaesth 2013;57:381-6.

4. Adamus $\mathrm{M}^{1}$, Jor O, Vavreckova T, Hrabalek L, Zapletalova J, Gabrhelik T, Tomaskova H, Janout V. Inter-observer reproducibility of 15 tests used for predicting difficult intubation. Biomed Pap Med Fac Univ Palacky Olomouc Czech Repub. 2011 Sep;155(3):275-81.

5. Khan ZH, Mohammadi M, Rasouli MR, Farrokhnia F, Khan RH. The diagnostic value of the upper lip bite test combined with sternomental distance, thyromental distance, and inter-incisor distance for prediction of easy laryngoscopy and intubation: A prospective study. Anesth Analg 2009;109:822-4.

6. Patil VU, Stehling LC, Zauder HL. Predicting the difficulty of intubation utilizing an intubation gauge. Anesthesiology Review 1983;X:32-3.

7. Cormack RS, Lehane J. Difficult intubation in obstetrics. Anesthesia 1984;39:1105-11.

8. Eberhart LH, Arndt C, Cierpka T, Schwanekamp J, Wulf H, Putzke C. The reliability and validity of the upper lip bite test compared with the Mallampati classification to predict difficult laryngoscopy: an external prospective evaluation. Anesth Analg 2005 Jul;101(1):284-9.

9. Shah PJ, Dubey KP, Yadav JP. Predictive value of upper lip bite test and ratio of height to thyromental distance compared to other multivariate airway assessment tests for difficult laryngoscopy in apparently normal patients. J Anaesthesiol Clin Pharmacol 2013 Apr;29(2):191-5.

10. Honarmand A, Safavi M, Yaraghi A, Attari M, Khazaei M, Zamani M. Comparison of five methods in predicting difficult laryngoscopy: Neck circumference, neck circumference to thyromental distance ratio, the ratio of height to thyromental distance, upper lip bite test and Mallampati test. Adv Biomed Res 2015 Jun 4;4:122.

11. Safavi M, Honarmand A, Zare N. A comparison of the ratio of patient's height to thyromental distance with the modified Mallampati and the upper lip bite test in predicting difficult laryngoscopy. Saudi J Anaesth. 2011 Jul; 5(3):258-63.

12. Myneni N, O'Leary AM, Sandison M, Roberts K. Evaluation of the upper lip bite test in predicting difficult laryngoscopy. J Clin Anesth 2010 May;22(3):174-8. 


\begin{tabular}{|c|l|}
\hline Term & \multicolumn{1}{c|}{ Description } \\
\hline True positive & A difficult laryngoscopy that had been predicted to be difficult \\
\hline False positive & An easy laryngoscopy that had been predicted to be difficult \\
\hline True negative & An easy laryngoscopy that had been predicted to be easy \\
\hline False negative & A difficult laryngoscopy that had been predicted to be easy \\
\hline Sensitivity & $\begin{array}{l}\text { The percentage of correctly predicted DVL as a proportion of all } \\
\text { laryngoscopies those were truly difficult. }\end{array}$ \\
\hline Specificity & $\begin{array}{l}\text { The percentage of correctly predicted EVL as a proportion of all } \\
\text { laryngoscopies those were truly easy. }\end{array}$ \\
\hline $\begin{array}{c}\text { Positive predictive } \\
\text { value }\end{array}$ & $\begin{array}{l}\text { The percentage of correctly predicted DVL as a proportion of all } \\
\text { predicted DVL. }\end{array}$ \\
\hline $\begin{array}{c}\text { Negative predictive } \\
\text { value }\end{array}$ & $\begin{array}{l}\text { The percentage of correctly predicted EVL as a proportion of all } \\
\text { predicted EVL. }\end{array}$ \\
\hline Accuracy & $\begin{array}{l}\text { It is the percentage of correctly predicted easy or difficult } \\
\text { laryngoscopies as a proportion of all laryngoscopies. }\end{array}$ \\
\hline \multicolumn{2}{|c|}{ Table 1: Statistical Terms and Definitions. ${ }^{\mathbf{b}}$} \\
\hline
\end{tabular}

EVL-Easy Visualisation of Larynx

DVL-Difficult Visualisation of Larynx

\begin{tabular}{|c|c|c|c|}
\hline Variable & EVL $(n=178)^{*}$ & DVL (n= 22) * & p value \\
\hline Gender & $\begin{array}{c}\text { Male- } 104 \\
\text { Female- } 74\end{array}$ & $\begin{array}{c}\text { Male-13 } \\
\text { Female- } 9\end{array}$ & Ns \\
\hline Age (years) & $27.99 \pm 5.76$ & $28.32 \pm 6.82$ & Ns \\
\hline Height $(\mathrm{cm})$ & $164.16 \pm 5.32$ & $162.26 \pm 5.25$ & Ns \\
\hline Weight (kg) & $59.25 \pm 5.84$ & $56.5 \pm 5.15$ & Ns \\
\hline BMI $\left(\mathrm{kg} / \mathrm{m}^{2}\right)$ & $21.95 \pm 1.66$ & $21.45 \pm 1.57$ & Ns \\
\hline ULBT & $\begin{array}{c}\text { Easy ( Class I \& II)-169 } \\
\text { Difficult (Class III)-9 }\end{array}$ & $\begin{array}{c}\text { Easy (Class I \& II)- } 6 \\
\text { Difficult (Class III) - } 16\end{array}$ & $<0.001$ \\
\hline TMD $(\mathrm{cm})$ & $6.75 \pm 0.05$ & $5.71 \pm 0.142$ & $<0.001$ \\
\hline SMD $(\mathrm{cm})$ & $13.37 \pm 0.21$ & $11.9 \pm 0.42$ & $<0.001$ \\
\hline IID (cm) & $4.808 \pm 0.44$ & $3.59 \pm 0.11$ & $<0.001$ \\
\hline
\end{tabular}

* The number mentioned against each parameter represents total numbers.

IID-Inter-incisor distance, Ns- non significant, SMD-Sternomental distance, TMD-Thyromental Distance, ULBT-Upper Lip Bite Test

\begin{tabular}{|c|c|c|c|c|}
\hline $\begin{array}{c}\text { Airway } \\
\text { Assessment Tests }\end{array}$ & $\begin{array}{c}\text { True Positive } \\
\text { (n) }\end{array}$ & $\begin{array}{c}\text { True } \\
\text { Negative } \\
\text { (n) }\end{array}$ & $\begin{array}{c}\text { False Positive } \\
\text { (n) }\end{array}$ & $\begin{array}{c}\text { False Negative } \\
\text { (n) }\end{array}$ \\
\hline ULBT Class III & 15 & 168 & 10 & 7 \\
\hline TMD $<6.5 \mathrm{~cm}$ & 14 & 160 & 18 & 8 \\
\hline SMD $<12.5 \mathrm{~cm}$ & 16 & 156 & 22 & 6 \\
\hline IID<4cm & 6 & 157 & 21 & 16 \\
\hline \multicolumn{6}{|r|}{ Table 3: The Performance of Different Airway Assessment Tests as } \\
Comparedto the Gold Standard (Direct Visualisation of Larynx) \\
\hline
\end{tabular}

IID-Inter-incisor distance, SMD-Sternomental distance, TMD-Thyromental distance, ULBT-Upper lip bite test.

\begin{tabular}{|c|c|c|c|c|c|}
\hline Tests & $\begin{array}{c}\text { Sensitivity } \\
(\%)\end{array}$ & $\begin{array}{c}\text { Specificity } \\
\text { (\%) }\end{array}$ & $\begin{array}{c}\text { Positive } \\
\text { Predictive Value } \\
(\%)\end{array}$ & $\begin{array}{c}\text { Negative } \\
\text { Predictive Value } \\
(\%)\end{array}$ & $\begin{array}{c}\text { Accuracy } \\
\text { (\%) }\end{array}$ \\
\hline ULBT & 68.1 & 94.3 & 60 & 96 & 91.5 \\
\hline TMD & 63.6 & 89.8 & 48.7 & 95.2 & 87.0 \\
\hline SMD & 72.7 & 87.6 & 42.1 & 96.3 & 86.0 \\
\hline IID & 27.2 & 88.0 & 22.2 & 90.7 & 81.5 \\
\hline ULBT + TMD & 45.5 & 99.4 & 90.9 & 93.6 & 94.0 \\
\hline ULBT + SMD & 50.0 & 99.4 & 91.6 & 94.1 & 93.5 \\
\hline ULBT + IID & 9.0 & 99.4 & 66.7 & 89.8 & 90.0 \\
\hline Table 4: Predictive Values of ULBT (Class III), TMD (<6.5cm), SMD (<12.5cm), IID (<4cm) \\
and their Combinations to Predict the Occurrence of Difficult Visualization of Larynx \\
\hline
\end{tabular}

IID-Inter-incisor distance, SMD-Sternomental distance, TMD-Thyromental distance, ULBT-Upper lip bite test. 